\title{
Instabilities on crystal surfaces: The two-component body-centered solid-on-solid model
}

\author{
Enrico Carlon and Henk van Beijeren \\ Institute for Theoretical Physics, University of Utrecht, P.O. Box 80006, 3508 TA Utrecht, the Netherlands \\ Giorgio Mazzeo \\ Dipartimento di Fisica, Università di Genova, and Istituto Nazionale per la Fisica della Materia, \\ via Dodecaneso 33, 16146 Genova, Italy
}

(Received 17 January 1996)

\begin{abstract}
The free energy of crystal surfaces that can be described by the two-component body-centered solid-on-solid model has been calculated in a mean-field approximation. The system may model ionic crystals with a bcc lattice structure (for instance $\mathrm{CsCl}$ ). Crossings between steps are energetically favored, which leads to the formation of networks of steps and of sharp edges between facets and rounded areas. There is a strong tendency to faceting even at finite temperatures, as seen experimentally in simple cubic ionic crystals $(\mathrm{NaCl})$. Transfer-matrix calculations corroborate these results. [S1063-651X(96)51306-1]

PACS number(s): 64.60.Cn
\end{abstract}

The shape of crystals in thermodynamic equilibrium can be found by minimizing the surface free energy at a given fixed volume. Usually this shape shows flat facets normal to low-index directions, in many cases connected to each other by rounded regions. Typically, at low temperatures, the rounded parts occupy a small portion of the total surface and the shape of the crystal is dominated by large flat areas; on increasing the temperature, the facets tend to shrink until they disappear from the equilibrium crystal shape (ECS) at a characteristic, facet-dependent temperature $T_{R}$, called the roughening temperature (see, for instance, [1]). Below $T_{R}$, when the facet is still present in the ECS, rounded regions either are connected smoothly to the facet, or they form sharp edges with the facet, when certain orientations become unstable and disappear from the ECS.

In this article we show how instabilities arise for certain orientations in the two-component BCSOS (body centered solid-on-solid) model. This model, which was shown before to yield a rich phase diagram [2] for the (001) crystal surface, describes as well a whole range of surface orientations of the form ( $t s 1$ ) with $|t|+|s| \leqslant 1$.

The bulk structure of the bcc crystal is composed of two different types of atoms $A$ and $B$ occupying the corners and the centers of an elementary cube, respectively. We consider a Hamiltonian of the form

$$
H=\frac{\epsilon}{2} \sum_{\langle i j\rangle}\left|h_{i}^{A}-h_{j}^{A}\right|+\frac{\epsilon^{\prime}}{2} \sum_{\langle k l\rangle}\left|h_{k}^{B}-h_{l}^{B}\right|,
$$

where surface configurations are given by a set of integer variables $h_{i}^{A}, h_{k}^{B}$ denoting the heights of the surface atoms with respect to a reference plane parallel to the (001) orientation. According to our convention $h_{i}^{A}$ and $h_{k}^{B}$ are odd and even integers, respectively. The $A$ and $B$ atoms at neighboring surface positions are constrained to have height difference \pm 1 . The couplings between atoms of the same species on next-nearest-neighbor positions ( $\epsilon$ for $A A$ and $\epsilon^{\prime}$ for $B B$, respectively) are taken to be negative and slightly different from each other. We define $\epsilon^{\prime}=\epsilon+2 \delta$, with $|\epsilon| \gg \delta>0$. This choice of parameters may be a reasonable approximation for certain ionic crystals where $A$ and $B$ are ions with opposite charge, interacting mainly through Coulombic forces. An example of such a crystal, with the desired lattice structure, is $\mathrm{CsCl}$.

The model can be mapped onto the six vertex model in a simple way [3]. Since the height differences between neighboring surface atoms assume only the values \pm 1 , the surface configurations can also be represented by arrows on the dual lattice, which may point in two opposite directions. The convention is that, seen from the arrow, the higher of the two neighboring surface atoms is at the right side. Uniqueness of the height variables restricts the vertex configurations to those six for which the ice rule holds: at every vertex there are two arrows pointing inwards and two outwards [Fig. 1(a)]. The energy defined by the Hamiltonian (1) can be reexpressed as a sum of vertex energies, defined as in Fig. 1(a) on two sublattices I and II, which alternate as the squares of different colors on a chess board.

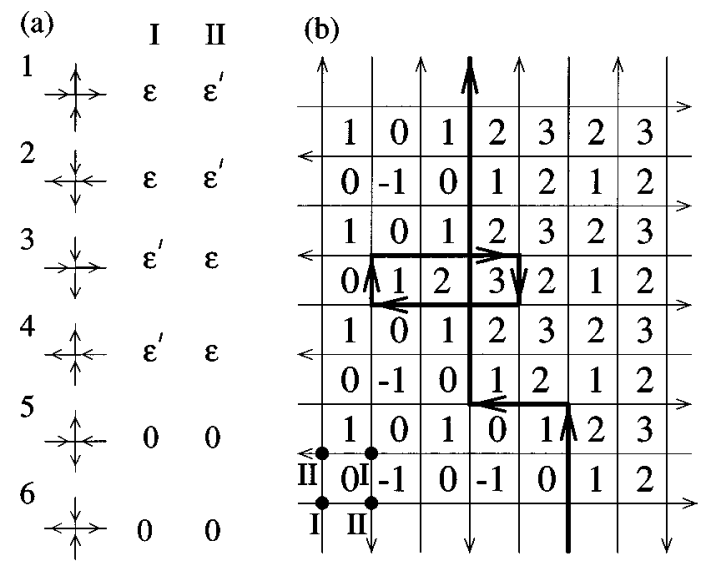

FIG. 1. (a) The six possible vertices and their energies on the two sublattices I and II, and (b) a surface configuration represented in terms of heights and vertices: the $A$ and $B$ atoms are always found at odd and even heights, respectively. Notice that the arrow directions make $45^{\circ}$ angles with the horizontal axes of the cubic lattice. 
The ground state of the model is twofold degenerate. In its vertex representation the arrows in subsequent columns or rows alternately point all up or all down, respectively, all right or all left, an arrangement known as the Manhattan lattice. The resulting surface is $c(2 \times 2)$ reconstructed, with one component $(B)$ at a constant height and the other $(A)$ with alternating heights above and below the first one. Figure 1(b) shows two elementary excitations of the ground state, a closed step and a step running between the boundaries of the system. A step is the boundary between two surface regions with a height difference of 2 (due to the inequivalence of the two sublattices no single-height step occurs). In the sixvertex representation it appears as a connected chain of reversed arrows. In reversing arrows one has to preserve the ice rule, which limits the possible trajectories. At a vertex site the step has two options: either to go straight, maintaining its previous direction, with an energy cost of $2 \delta$, or to turn opposite to the direction of an arrow on the Manhattan lattice, which costs an energy $-\epsilon$. If at a given vertex all arrows are reversed, we interpret this as the crossing of two steps, rather than two steps touching at a corner. The reason is that corners are very rare, because of their high energy, whereas the crossing of two steps costs no energy: on the contrary, one regains the energy $4 \delta$ that would have to be invested if the steps would not cross.

Our analysis can be divided into two parts.

(A) First we evaluate the free energy of a single isolated step. The step partition function is approximated with that of a random walk on the Manhattan lattice with weight $e^{-2 \beta \delta}$ for each straight segment and $e^{\beta \epsilon}$ for each turn. At low temperatures, when $e^{\beta \epsilon} \ll e^{-2 \beta \delta}$, steps consist of elongated segments with only rare turns and self-correlations can be neglected. We also neglect interactions with closed steps [such as the one shown in Fig. 1(b)], since those give a relevant contribution to the single-step free energy only if present at a reasonable density. A comparison with numerical results will give good indications regarding the range of validity of these approximations.

The single-step free energy per unit of length $f_{s}(\phi)$, obtained in the Manhattan walk approximation, is shown in Fig. 2(a) (solid lines), for walks making an angle $\phi=0$ or $\phi=\pi / 4$ with one of the main axes of the vertex lattice. The dotted lines are finite size results obtained from transfermatrix (TM) calculations, giving exact numerical values of step free energies on strips of size $N \times \infty$, shown for some values of $N$ in the figure. At low temperatures, TM data extrapolate very well towards the values calculated with the random-walk model. Close to the roughening temperature the random-walk approximation fails, because the interactions with closed steps become important, whereas the TM results extrapolate to the correct exponentially vanishing behavior of the step free energy [1].

(B) Next we evaluate the free energy of a collection of interacting steps, or equivalently the free energy of an orientation tilted with respect to the reference (001) facet (as done above we neglect closed loops of reversed arrows). The polarizations of the vertex lattice, $p=n_{\uparrow}-n_{\downarrow}, q=n_{\rightarrow}-n_{\leftarrow}$ ( $n_{\uparrow}, n_{\downarrow}, n_{\rightarrow}$, and $n_{\leftarrow}$ are the densities of up, down, right, and left arrows) are related to the slope parameters $t$ and $s$ of the surface by the relationships $t=(q-p) / 2 ; s=(q+p) / 2$. (a)

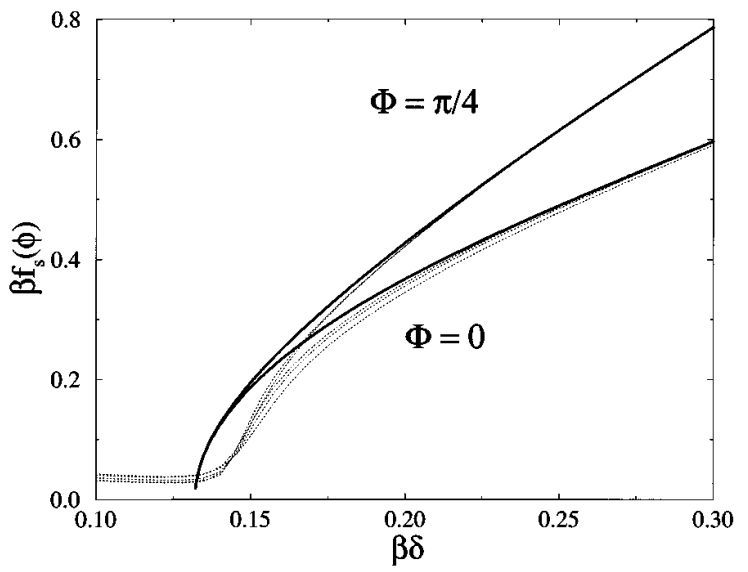

(b)

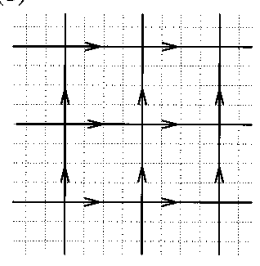

(c)

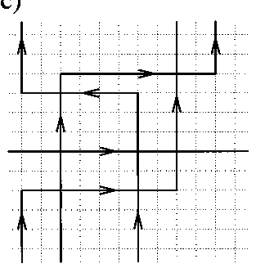

(d)

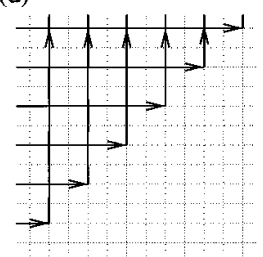

FIG. 2. (a) $\beta f_{s}(\phi)$ for steps of two different orientations as function of $\beta \delta$ and with $\epsilon / \delta=-10$. The solid lines are calculations done using the random-walk model, while the dotted lines are obtained from TM techniques. Possible patterns of steps at $T=0$ (b) and at finite values of $T$ (c); a faceted surface at $T=0$ with a (011) and a (001) facet joining at a sharp edge (d). For convenience we have drawn only the reversed arrows on the Manhattan background.

At $T=0$ and fixed $p$ and $q$ a surface configuration is generated by a network of perfectly straight horizontal and vertical steps. Turns are not allowed [Fig. 2(b)]. For homogeneous step densities $p$ and $q$ the ground-state energy per unit projected area is given by

$$
\varepsilon_{0}(p, q)=\delta(|q|+|p|)-\delta|q p|
$$

The linear terms represent the contribution of isolated vertical and horizontal steps forming the network, while the quadratic term is due to the decrease of energy at the crossings. This form for the ground-state energy is a nonconvex function of $p$ and $q$, implying that a uniform network of steps is unstable with regard to faceting, in other words, to phase separation of an unstable surface orientation into stable ones [1]. A stable configuration for the case $p=q$ is illustrated in Figure 2(d). It consists of a (011) and a (001) facet joining at a sharp edge. Figure 2(c) shows a possible configuration of steps at nonzero temperature. If the temperature is sufficiently low the free energy as a function of $p$ and $q$ remains concave for some range of values for $p$ and $q$, which will lead to faceting again, with the disappearance of some crystal orientations from the ECS.

We calculated $f(p, q)$, the free energy of a surface of given orientation $p$ and $q$ (per unit of projected area), under the assumption that this orientation is generated by a homogeneous density of steps forming an angle $\phi=\arctan (p / q)$ 
(a)

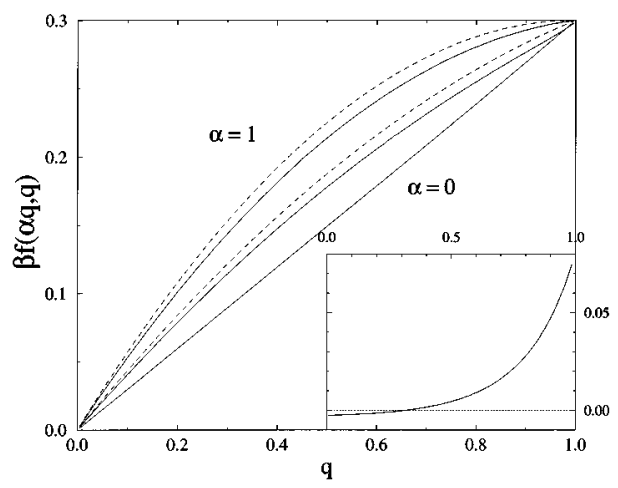

(b)

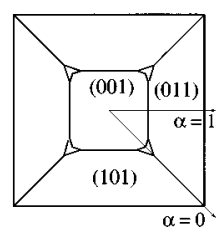

FIG. 3. (a) Surface free energies per unit of projected area at $\beta \epsilon=-3$ and $\beta \delta=0.3$ and for $\alpha=0,1 / 2,1$ (solid lines). Groundstate energies given by Eq. (2) for $\alpha=1 / 2,1$ (dashed lines). In the inset: the second derivative of $f(q)$ along the line $\alpha=0$. (b) The corresponding ECS (seen from above) has a tiny rounded area around the corner between the (001), (101), and (011) facets.

with the vertical axis of Fig. 1(b). In a mean-field approximation the total free energy is equal to the free energy of a step in a "background" of other steps $\left[\widetilde{f}_{s}(\phi)\right]$ times the step density:

$$
f(p, q)=\frac{\sqrt{p^{2}+q^{2}}}{2} \widetilde{f}_{s}(\phi),
$$

where $\widetilde{f}_{s}(\phi)$ has been calculated in the Manhattan randomwalk approximation, taking into account that the step has a certain probability of intersecting other steps (the details will appear in a longer publication [4]). Equation (3) reduces to Eq. (2) in the limit $T \rightarrow 0$.

Figures 3 and 4 show the free energies (per unit of projected area) for different orientations, calculated at three temperatures. The curves are plotted as a function of the vertical polarization $q$ at a constant ratio $p / q=\alpha$ [the correspondence between $\alpha$ and the crystallographic axes is shown in Fig. 3(b)]. As can be shown easily, for $q=1$ and irrespective of the value of $p$, the exact value of the free energy in the thermodynamic limit is simply equal to $\delta$. This is reproduced correctly by our mean-field calculations. As indicated before, we expect these results to be best at low temperatures and large values of $|\epsilon| / \delta$.

The two upper solid lines of Fig. 3(a) are the mean-field free energies calculated along the lines $\alpha=1,1 / 2$. The two dotted lines are the values of the ground-state energy. The free energy along $\alpha=0$, which at $T=0$ is a straight line [Eq. (2)], becomes concave at small $q$, while it is convex at larger values of $q$ [see inset of Fig. 3(a)]. The ECS at this value of the temperature is shown in Fig. 3(b): only a small area at the corner formed by the (001), the (011), and the (101) facet is rounded.

At higher temperatures the rounded area in the ECS becomes larger. The orientations along the line $\alpha=1$ are still all unstable for $\beta \epsilon=-2.0$ and $\beta \delta=0.2$, so the (001) and the (011) facet are still joined directly. At $\beta \epsilon=-1.5$ and $\beta \delta=0.15$ the two facets are separated by a rounded region. For all temperatures analyzed we find a region of unstable (a)

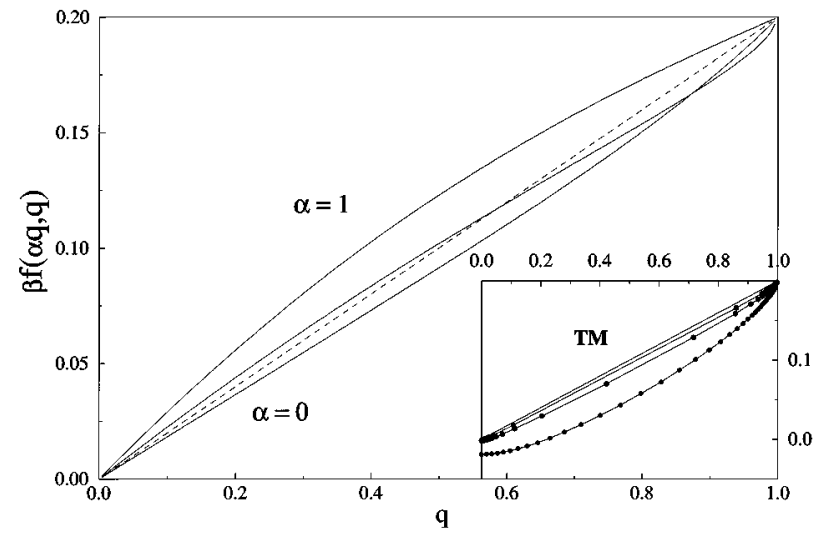

(b)

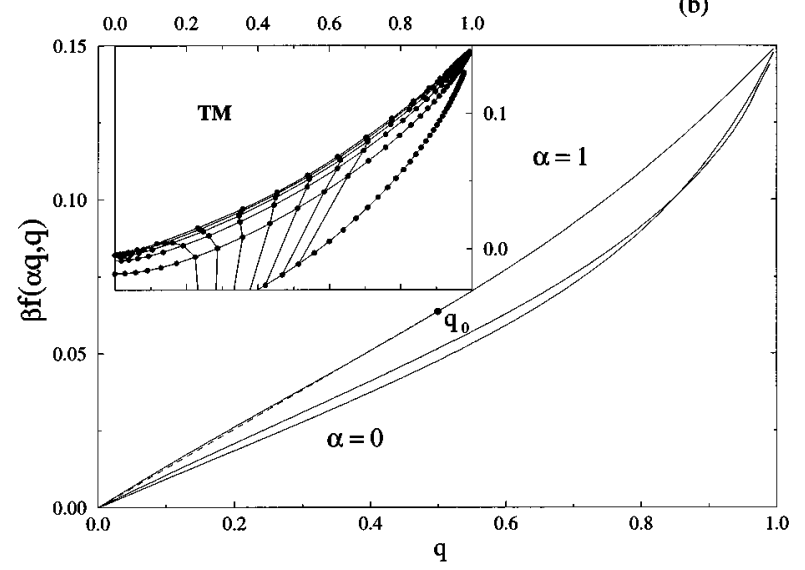

FIG. 4. As in Fig. 3(a) at higher temperatures: (a) $\beta \epsilon=-2$ and $\beta \delta=0.2$ and (b) $\beta \epsilon=-1.5$ and $\beta \delta=0.15$. The dashed line in (a) is a straight line, as guide for the eye. In (b) all the orientations in the range $0 \leqslant q \leqslant q_{0} \approx 0.5$ along the line $\alpha=1$ are unstable, as shown by the Maxwell construction. In the insets: TM calculations on finitesize systems performed along the line $\alpha=1$, where the lowest curves correspond to the smallest sizes. In the inset to (b) points measured at equal values of $h$ are connected along the different curves $f_{N}(q)$.

vicinal orientations surrounding the (001) facet. This implies the existence of a sharp ridge between this facet and the rounded regions surrounding it, which are caused by the competition between attractive and repulsive step-step interactions. Attractions are due to the energy gained at crossings and would favor a total phase separation between the facets like at $T=0$ [see Eq. (2)]. Repulsions are due to entropic effects: steps cannot occupy the same bonds of the vertex lattice and their entropy is reduced when they are brought together [5]. At low step densities attractions dominate, while entropic repulsions become effective at higher step densities with the effect of stabilizing the surface free energy.

As to the connections between the (011) and (101) facet: for $q$ near unity and $\alpha$ near zero our mean-field calculations yield a concave $f(p, q)$ along lines of constant $q$. This suggests a sharp ridge between the facets, extending into a ridge between rounded areas and ending in a critical point. There would be no conical point, as described in Ref. [6]. This, 
however, may well be due to the mean-field approximation used and deserves further investigation.

We performed TM calculations to check the predictions of the mean-field theory. External fields $h$ and $v$ (the variables that are conjugate to $p$ and $q$ ), may be used to produce orientations tilted with respect to the (001) facet. Choosing $h=v$ we generated all the orientations $(0 s 1)$ from (001) to (011) along $\alpha=1$. We calculated $\hat{f}_{N}(h)$, the free energy as a function of the applied field, and its Legendre transform $f_{N}(q)$ for an $N \times \infty$ strip. The latter is nothing but the free energy as function of the polarizations for $p=q$ [7]. It can be directly compared with the mean-field results in the limit $N \rightarrow \infty$. The insets of Figs. 4(a) and 4(b) show $f_{N}(q)$ vs $q$ measured at equally spaced values of $h$ (circles); no instabilities show up for finite systems, and the curves $f_{N}(q)$ indeed are always convex. Yet the trend towards phase separation can be observed, by considering the scaling behavior with $N$. In the inset of Fig. 4(a), for large sizes, all the points tend towards the values $q=p=0$ and $q=p=1$, which according to our mean-field results are the only stable orientations. In the inset of Fig. 4(b) instead the points seem to separate partially between $q=0$ and around $0.4 \leqslant q \leqslant 0.6$. Unfortunately, the largest size accessible to our numerical calculations $(N=12)$ is not large enough to allow a detailed finite-size scaling of the TM data, but the existence of partial phase separation is qualitatively confirmed.

In conclusion, we have evaluated free energies of surfaces vicinal to the (001) facet in the two-component BCSOS model. Mean-field calculations show phase separation (depending upon temperature) between the (001) facet and either the (011) facet or rounded side orientations, with a sharp edge at the facet boundary in either case. A similar feature has been suggested before for a different model [8], but was abandoned later [9] as a possible artifact of the mean-field approximation. Our TM results make us quite confident that the sharp edge is not an artifact in the present case [10]. Our model follows quite closely the thermal evolution found experimentally in $\mathrm{NaCl}$ [11] and investigated theoretically $[8,12]$ in models for ionic crystals of the rocksalt type. Although there are some differences, which will be discussed in more detail elsewhere [4], the common features are a strong tendency towards faceting even at finite temperatures.

We thank D.J. Bukman, J.W.M. Frenken, and J.D. Shore for valuable comments and discussions; G.M. was supported, in part, by grant No. ERBCHBGCT920197 from the EC Human Capital and Mobility Programme.
[1] H. van Beijeren and I. Nolden, in Structure and Dynamics of Surfaces, edited by W. Schommers and P. von Blanckenhagen (Springer-Verlag, Berlin, 1987), Vol. 2, pp. 259-300.

[2] G. Mazzeo, E. Carlon, and H. van Beijeren, Phys. Rev. Lett. 74, 1391 (1995).

[3] H. van Beijeren, Phys. Rev. Lett. 38, 993 (1977).

[4] E. Carlon and H. van Beijeren (unpublished).

[5] E. E. Gruber and W. W. Mullins, J. Phys. Chem. Solids 28, 875 (1967).

[6] D. J. Bukman and J. D. Shore, J. Stat. Phys. 78, 1277 (1995).

[7] A. F. Andreev, Zh. Eksp. Teor. Fiz. 80, 2042 (1980) [Sov. Phys. JETP 53, 1063 (1981)].
[8] C. Rottman and M. Wortis, Phys. Rev. B 29, 328 (1984).

[9] M. Wortis, in Chemistry and Physics of Solid Surfaces VII, edited by R. Vanselow and R. F. Howe (Springer-Verlag, Berlin, 1988), pp. 367-405.

[10] It should be stressed here that the (001) orientation is reconstructed and that TM results [4] show that stable tilted orientations are unreconstructed. The competition between reconstructed and unreconstructed orientations may lead to sharp edges in the ECS, as shown, for instance, in E. D. Williams, Surf. Sci. 299/300, 502 (1994).

[11] J. C. Heyraud and J. J. Métois, J. Cryst. Growth 84, 503 (1987).

[12] J. D. Shore and D. J. Bukman, Phys. Rev. E 51, 4196 (1995). 